\title{
O Ensino de Programação com Scratch e seu Impacto na Opção Profissional para Meninas
}

\author{
Francielle de Mattos, Vinicius Ferreira, Junia Anacleto \\ Departamento de Computação - Universidade Federal de São Carlos (UFSCar) \\ Caixa Postal 676 - 13.565-905 - São Carlos - SP - Brasil \\ \{francielle.mattos, vinicius.ferreira, junia.anacleto\}@dc.ufscar.br
}

\begin{abstract}
With the rise of computing area in the labor market, few female professionals are noticed in this area. To promote gender equality, an exploited approach is the programming education. Once programming education is still challenging for the area of Computer Education, Scratch programming language has emerged as a promising proposal. In this context, this article aims to analyze studies using Scratch as a tool to encourage girls who are defining their career. A systematic mapping was conducted to identify what approaches have been used. As a result, there was found a number of initiatives, but few studies have been conducted, especially in relation to the effectiveness of the pedagogical approaches.
\end{abstract}

Resumo. Com a ascensão da computação no mercado de trabalho, observa-se poucas profissionais do sexo feminino nessa área. Para fomentar a igualdade de gênero, uma abordagem explorada é do ensino de programação. Como o ensino de programação ainda é desafiador para a área de Educação em Computação, o Scratch surgiu como uma proposta promissora. Assim, este artigo analisa estudos que utilizaram o Scratch como ferramenta para incentivar garotas que estão em período de definição profissional. Foi realizado um mapeamento sistemático para apontar as abordagens que têm sido utilizadas. Como resultado, verificou-se várias iniciativas, mas poucos estudos sendo realizados, principalmente, em relação à eficácia das abordagens pedagógicas.

\section{Introdução}

A crescente demanda por novas soluções tecnológicas tem impulsionado o mercado e o campo da computação. Porém, no mercado de trabalho, este é um território dominado por profissionais do sexo masculino [Maia 2016]. Sendo que a escolha pela futura profissão é desenvolvida durante o período escolar [Krendl et al. 1989]. Assim, verifica-se a necessidade de apresentar para os alunos, principalmente para as mulheres do ensino básico, os conhecimentos básicos de computação e programação.

O ensino de programação nas escolas tem crescido no mundo [Saeli et al. 2011]. Não obstante, no Brasil várias escolas estão incorporando isso em suas atividades curriculares. Aprender programação é importante para estimular a criatividade, autonomia e desenvolver o raciocínio lógico, bem como a resolução de problemas e o promover o trabalho em equipe. Tais habilidades são cruciais na formação do indivíduo e altamente valorizadas pelo mercado de trabalho. Além disso, com a onipresença das Tecnologias da Informação e Comunicação (TICs) na sociedade atual, estar ciente dos 
V Congresso Brasileiro de Informática na Educação (CBIE 2016)

Anais do XXVII Simpósio Brasileiro de Informática na Educação (SBIE 2016)

usos e dos conceitos práticos da computação é extremamente relevante ${ }^{1}$. Nesse contexto, visando facilitar a introdução aos conceitos de computação e a programação para crianças e jovens, surgiu em 2007 o Scratch.

Scratch $^{2}$ é uma linguagem de programação, criada no Media Lab do MIT, que possibilita a criação de aplicações, jogos, histórias e animações, de forma mais acessível por meio de uma interface gráfica intuitiva. Assim, para programar, os comandos devem ser agrupados de maneira lógica, como se fossem blocos de montar Lego. Os comandos são divididos em categorias e podem ser facilmente encaixados e encadeados de forma a produzirem as ações desejadas. A linguagem está disponível em mais de 40 idiomas e permite uma forma de interação mais simples, lúdica e intuitiva, diferenciando-se de outras linguagens e plataformas como o Alice, Game Maker, Kodu e Greenfoot. Essas vantagens podem influenciar jovens a desenvolverem habilidades de programação e assim decidir seguir uma carreira na área de computação.

O interesse das mulheres por ciência da computação e áreas afins é perdido desde muito cedo em sua formação. Enquanto os meninos são, em muitos casos, incentivados a explorar desde criança os meios tecnológicos sem permissão explícita, as meninas da mesma faixa etária, muitas vezes, necessitam de permissão para utilizar computadores sozinhas [Krendl et al. 1989]. Por mais que as salas de tecnologia nas escolas têm aumentado, observa-se, em certos casos, um monopólio dos meninos com o instrutor durante as aulas buscando mostrar e aprimorar seu conhecimento prévio. Isso tem influenciado o aprendizado das meninas e colaborado na construção da ideia em que a área de computação é de domínio masculino [Huber e Scaglion 1995]. Nesse cenário dominado pelo sexo masculino, as mulheres se sentem meras coadjuvantes e menos confortáveis nas áreas relacionadas à computação. Tal estigma pode fazer com que as mulheres se sintam menos capacitadas para atuar nessas áreas. Por isso, o acesso e exposição igualitário de experiência e conhecimento computacional durante o período escolar é imprescindível para contornar esse paradigma.

Como o ensino de programação é um grande desafio na área de Educação em Computação, pretende-se analisar como a linguagem de programação Scratch tem sido utilizada como ferramenta de ensino-aprendizagem para ensinar programação para incentivar garotas a perseguir uma carreira na área de computação. Diante disso, os autores do presente artigo consideraram importante mapear os estudos realizados nessa área para construir um cenário. Com isso, espera-se ter uma visão ampla dessas publicações para compreender as tendências e deficiências, para assim incentivar outros estudos pertinentes à área.

\section{Mulheres na Computação}

Embora a hierarquia entre os sexos seja um problema histórico, suas consequências e reprodução ainda persistem em nossa sociedade moderna. Entretanto, essa hierarquia pautada na diferenciação biológica está sendo descontruída e superada aos poucos. O problema da "divisão sexual do trabalho" tem diversas consequências como estigmatização da mulher para a esfera reprodutiva e doméstica. Além disso, designa aos

\footnotetext{
${ }^{1}$ Revista Escola. Por que ensinar programação na escola. 2016 Acessado em Maio/2016. http://revistaes cola.abril.com.br/blogs/tecnologia-educacao/2016/02/23/por-que-ensinar-programacao-na-escola/

${ }^{2}$ Scratch. 2016. Acessado em Maio/2016. https://scratch.mit.edu.
} 
V Congresso Brasileiro de Informática na Educação (CBIE 2016)

Anais do XXVII Simpósio Brasileiro de Informática na Educação (SBIE 2016)

homens prioritariamente os cargos de alto valor e prestígio [Maia 2016]. Sendo que a capacitação é construída e aperfeiçoada durante a formação do profissional. Para Lombardi (2006), o equilíbrio sexual no mercado de trabalho é benéfico para áreas de atuação. Assim, a autora verificou que o fortalecimento da presença feminina nos cursos de engenharia teve impacto na inserção das mulheres no mercado de trabalho. Com isso, os estereótipos de gênero foram perdendo seu valor. No entanto, para Maia (2016) esses estereótipos ainda persistem na computação e áreas afins.

Para Gürer e Camp (2002), em sua revisão da literatura visando entender o fenômeno em relação à carência de mulheres na computação, verificaram que diversos fatores estão relacionados ao fenômeno da ausência de mulheres na computação. As faltas de incentivo e igualdade de acesso para explorar tecnologias desde as fases iniciais de vida, exposição a atitudes positivas em relação à profissão de computação, oportunidade para desenvolver habilidades com tecnologias, mentoria e autoconfiança são alguns dos motivos que desestimulam as mulheres a seguirem carreiras na computação. Outros fatores são o ambiente hostil criado pelos homens, influências sociais como a ideia negativa de que computação é uma área somente para nerds ou homens.

De acordo com Gordon (1981), a falta de planejamento para o desenvolvimento de carreiras entre os alunos é um grande complicador nas escolas. Isso afeta principalmente os alunos do ensino médio, pois eles estão em processo de transição da escola para a universidade e precisam definir uma carreira. Para Wulff e Steitz (1999), fatores como autoeficácia e autoestima estão diretamente relacionados à indecisão de carreira entre meninas do ensino médio.

Nesse contexto, mudar esse paradigma é importante para construir uma sociedade com melhor igualdade de oportunidades para mulheres e homens. Além disso, ter mulheres na computação implica ter olhares para outros problemas que não compõe comumente o universo masculino.

\section{Revisão da Literatura}

Com o objetivo de revisar na literatura publicações que relatam o uso de Scratch para incentivar meninas no Ensino Médio para a área de computação, neste artigo foi utilizado o processo de mapeamento sistemático. Para um maior rigor na execução do método, foram consideradas as diretrizes formalizadas por Petersen et al. (2008) e Kitchenham et al. (2009). Similarmente a revisão sistemática, um mapeamento sistemático segue uma estratégia pré-definida, porém flexível, visando responder a uma ou mais questões de pesquisa de forma imparcial e replicável [Petersen et al. 2008; Kitchenham et al. 2009]. Para apoiar a execução de todo o processo de mapeamento sistemático foi utilizado a ferramenta StArt. Essa ferramenta torna mais ágil, preciso, replicável e organizado a realização de revisões e mapeamentos sistemáticos [Zamboni e Fabbri 2010].

Este mapeamento sistemático visa responder as seguintes questões de pesquisa:

- RQ1 - Quantas publicações relatam que o uso do Scratch, como ferramenta, para incentivar alunos do ensino médio para a iniciação à programação?

- RQ2 - Quais dessas publicações visam incentivar mais mulheres para área da Ciência da Computação?

- RQ3 - Quais abordagens de ensino são descritas nessas publicações? 
V Congresso Brasileiro de Informática na Educação (CBIE 2016)

Anais do XXVII Simpósio Brasileiro de Informática na Educação (SBIE 2016)

\subsection{Estudo piloto}

Visando ter um panorama do tema, decidiu-se realizar um estudo piloto. Para Mackey e Gass (2005), um estudo piloto ou preliminar é um considerado um estudo exploratório e em pequena escala que permite familiarizar-se com o escopo do estudo e assim testar, avaliar, revisar e aprimorar os instrumentos e procedimentos de pesquisa. Esse estudo preliminar foi conduzido de forma incremental e interativa, em três etapas.

$\mathrm{Na}$ primeira etapa, o objetivo era explorar o tema de forma abrangente para verificar e definir as bases científicas a serem investigadas. Baseando-se nas bases suportadas pela ferramenta StArt, foi construída a String 1 (ver tabela 1) para buscar publicações que tratavam do ensino de computação nas escolas. Para eliminar falsospositivos e estudos irrelevantes para essa proposta, os resultados foram limitados para somente busca no resumo, título ou palavras-chave e áreas relacionadas a Ciência da Computação e Educação. Com isso, chegou-se ao número de mais de 21.458 publicações. Isso demonstra o grande interesse da comunidade acadêmica no assunto. Além disso, analisou-se um crescimento nos últimos anos no tema, evidenciando que o tema é vasto e ainda há muito a ser explorado e validado. Como resultado dessa etapa, definiu-se como relevante as seguintes bases: ACM, IEEE, Science Direct, SCOPUS e Springer.

String 1 - ("computer science" OR computing OR programming) AND (learn OR learning OR education OR teaching OR training) AND (school OR “ $K-12$ ”)

String 2 - scratch AND ("computer science” OR computing OR programming) AND (learn OR learning OR education OR teaching OR training) AND (school OR "K-12”)

String 3 - scratch AND ("computer science” OR computing OR programming) AND (learn OR learning OR education OR teaching OR training) AND ("middle school" OR "high school" OR "secondary school")

Tabela 1. Strings de busca utilizadas no estudo piloto

Com o surgimento do Scratch em 2007, como uma proposta promissora para o ensino de leigos em programação, na segunda etapa buscou-se nas bases de conhecimento, definidas na etapa anterior, publicações que exploram o ensino de Scratch nas escolas. Para isso, adicionou-se o termo Scratch a string de busca e limitou-se para somente artigos posteriores à 2007. Com isso, foram encontradas 1.153 publicações.

Sabendo do problema de indecisão na escolha de profissão que atinge os jovens, principalmente os estudantes do ensino médio, na terceira etapa foi construída a String 3. Esse problema foi evidenciado por uma pesquisa ${ }^{3}$ com dois mil jovens do terceiro ano do ensino médio no Brasil. Esse estudo mostrou que mais da metade desses jovens não sabe qual carreira seguir, evidenciando a falta de orientação vocacional nas escolas. Nessa etapa foram encontradas 601 publicações, respondendo assim a RQ1. A figura 1 mostra a evolução e tendência do uso do Scratch no ensino médio nas escolas. Além disso, as publicações têm sido em sua maioria publicadas em conferências, se destacando a ACM Technical Symposium on Computer Science Education com 45 publicações. Analisando a tabela 2 e verificando-se os resultados da terceira etapa, notou-se que as publicações indexadas pela Scopus eram em sua maioria as mesmas da ACM. Sendo assim, foram encontradas 47 publicações duplicadas.

\footnotetext{
${ }^{3}$ Escolha profissional: a indecisão do jovem frente aos desafios da carreira. Junho/2016. http://portalde jornalismo-rj.espm.br/escolha-profissional-a-indecisao-do-jovem-frente-aos-desafios-da-carreira/
} 
V Congresso Brasileiro de Informática na Educação (CBIE 2016)

Anais do XXVII Simpósio Brasileiro de Informática na Educação (SBIE 2016)
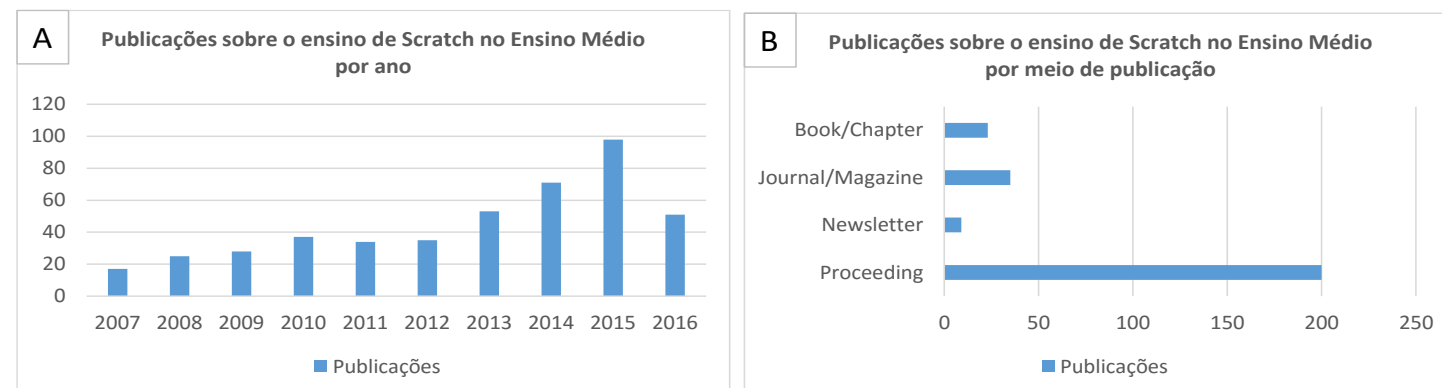

Figura 1. Publicações sobre o ensino de Scratch no Ensino Médio (a) por ano e (b) por meio de publicação

\begin{tabular}{|c|c|c|c|}
\hline \multirow{2}{*}{ Base } & \multicolumn{3}{|c|}{ Publicações encontradas } \\
\cline { 2 - 4 } & Etapa 1 & Etapa 2 & Etapa 3 \\
\hline ACM & 6.464 & 121 & 66 \\
\hline IEEE & 78 & 7 & 7 \\
\hline Science Direct & 1.888 & 13 & 65 \\
\hline Scopus & 8.383 & 145 & 457 \\
\hline Springer & 4.645 & 867 & 601 \\
\hline Total & 21.458 & 1.153 & 6 \\
\hline
\end{tabular}

Tabela 2. Publicações encontradas com o estudo piloto

\subsection{Buscas de estudos primários}

A partir da definição dos objetivos, questões de pesquisa e do estudo piloto, foi elaborada a seguinte string de busca para buscar publicações que relatassem o uso do Scratch para incentivar mulheres na computação:

scratch AND ("computer science" OR computing OR programming) AND (learn OR learning OR education OR teaching OR training) AND ("middle school" OR "high school" OR "secondary school") AND (girl OR girls OR woman OR women OR female OR lady OR ladies)

Segundo Kitchenham et al. (2009), o processo de busca deve contemplar a obtenção uma grande variedade de estudos conhecidos. Para complementar o processo de busca automático nas bases definidas no estudo piloto, foi adotado o método de snowballing como estratégia para buscar recursivamente publicações que não foram recuperadas no processo de busca, mas que podem ser interessantes para o estudo [Wohlin 2014].

\subsection{Critérios de inclusão e exclusão}

Afim de decidir quais publicações seriam úteis para o mapeamento, foram definidos os critérios de inclusão: Artigos primários que abordem um estudo de caso sobre o uso do Scratch no Ensino Médio com intuito de incentivar meninas para a área de computação. Como critérios de exclusão, definiu-se que seriam excluídos publicações que não eram artigos completos peer-reviewed, ou que não estejam disponíveis por meio eletrônico, ou que não se enquadram nos idiomas definidos, ou artigos que não reportem o uso do Scratch com intuito de incentivar meninas à área de computação.

Os critérios de inclusão e exclusão foram aplicados em duas etapas. Primeiramente, os artigos foram julgados com base na análise de título e resumo. Na segunda etapa, os artigos foram avaliados com base na leitura da introdução, metodologia e conclusão de cada estudo. Esse processo foi realizado por dois pesquisadores de forma independente e as discordâncias eram resolvidas por meio de um consenso. 
V Congresso Brasileiro de Informática na Educação (CBIE 2016)

Anais do XXVII Simpósio Brasileiro de Informática na Educação (SBIE 2016)

\subsection{Extração de dados}

Definiu-se os seguintes dados para serem extraídos: i. Atividade realizada e o público-alvo; ii. Tipo de abordagem de ensino; iii. Resultados; iv. Lições aprendidas.

\subsection{Resultados das buscas}

Após a fase inicial do processo de seleção, foram encontrados 242 artigos. Desses artigos, 228 foram excluídos pois eram duplicados ou foram considerados irrelevantes para o objetivo. O resultado final dessa primeira etapa pode ser observado na Tabela 3.

\begin{tabular}{|l|c|c|c|c|c|c|}
\hline & ACM & IEEE & Science Direct & SCOPUS & Springer & Total \\
\hline Artigos incluídos & 5 & 0 & 0 & 0 & 9 & 14 \\
\hline Artigos excluídos & 3 & 0 & 0 & 7 & 218 & 228 \\
\hline
\end{tabular}

Tabela 3. Publicações incluídas e excluídas por base, levando em consideração apenas, título, resumo e palavras-chave

Dos artigos excluídos, 147 não estavam acessíveis, 14 eram publicações anteriores a 2007, 7 eram duplicados e 50 não atendiam os critérios de inclusão, como por exemplo ser um artigo completo ou não eram estudos de caso. Após o levantamento prévio, foi realizado um refinamento dos artigos analisando a introdução, metodologia e conclusão dos artigos, assim dos 14 artigos apenas 5 foram considerados relevantes e aceitos nos critérios de inclusão. Aplicando-se a técnica de snowballing nesses artigos não foram encontrados novos artigos.

\subsection{Discussões}

A partir da leitura completa dos artigos, foram extraídos dados e verificadas as tendências para encontrar evidências que possam sugerir futuros estudos. Nesta seção, apresentamse resumidamente os dados, a análise e discussão dos mesmos. O objetivo foi compreender quais abordagens os autores estão utilizando para incentivar meninas para seguirem carreira na computação e áreas afins.

Mota e Adamatti (2015), como parte do projeto "Meninas e Jovens Fazendo Ciências Exatas, Engenharias e Computação", realizaram um workshop para ensinar de lógica computacional utilizando Scratch para alunos do ensino médio de uma escola pública no Brasil, que não tinham conhecimento prévio em programação. Após a realização do curso, foi aplicado um questionário discursivo, para identificar o conhecimento em computação, a motivação e dificuldades no aprendizado, e pensamentos sobre as diferenças de gêneros na carreira de computação. O desinteresse demonstrado pelas meninas no início foi superado ao longo do workshop. Durante a análise dos questionários, indícios mostraram que questões culturais sobre a diferenciação sexual e biológica estão relacionadas ao desinteresse das mulheres pela área da computação. Além disso, alguns estudantes relataram que mulheres são mais sociais e por isso são melhores em áreas de "cuidado" como saúde e educação.

Webb e Rosson (2013), realizaram um workshop de Scratch durante quatro dias para 15 meninas durante as férias escolares. As atividades realizadas utilizaram a abordagem de ensino "scaffolding", onde a dificuldade das atividades são elevadas progressivamente com o intuito de despertar uma maior independência do aluno. No workshop as meninas tinham aulas e como atividades práticas precisavam investigar e corrigir problemas em exemplos pré-concebidos em Scratch. Durante o curso foi 
V Congresso Brasileiro de Informática na Educação (CBIE 2016)

Anais do XXVII Simpósio Brasileiro de Informática na Educação (SBIE 2016)

verificado que a maioria das meninas possuíam certo conhecimento com Scratch e algumas relataram já ter utilizado ferramentas computacionais como Alice e Lego Robotics. Todos os dias as meninas eram entrevistadas para verificar o conhecimento adquirido. Os autores verificaram um aumento na taxa de acerto nas atividades entre o primeiro e o último dia. Além disso, a abordagem teve um impacto positivo no engajamento das meninas, aumentando o interesse para programação e criação de projetos próprios.

Feldhausen et al. (2014) utilizaram a linguagem Scratch para ensinar conceitos de computação paralela. Os autores realizaram dois workshops, para 40 meninas do ensino fundamental/médio e outro para alunos iniciantes do curso de graduação em ciência da computação. Usando o ambiente de desenvolvimento do Scratch e comparando ambos cenários, os alunos com pouco ou nenhum conhecimento de programação foram capazes de construir, modificar e observar as mudanças no desempenho das aplicações e operações multitarefas. Os autores verificaram em questionário posterior ao curso que as meninas se sentiram motivadas e capazes de criar programas de computador. Além disso, 22 garotas demonstraram interesse em trabalhar com computação.

Konak (2016) criou um workshop de um dia para ensinar alunos do ensino médio, principalmente meninas, a programar usando Scratch. Para auxiliar no ensino, alunos de uma universidade atuaram como tutores. Durante o workshop, os alunos eram incentivados a construir um mini-projeto com as técnicas que haviam aprendido e depois foram convidados para criar um jogo. Nenhum dos estudantes tinham utilizado anteriormente Scratch. Antes do workshop, a maioria das meninas mostravam pouco interesse pela carreira em computação ao contrário da maioria dos meninos. Os meninos no início pensavam que tinham melhores habilidades com o computador do que as meninas, porém, no final do workshop, as meninas criaram os jogos mais interessantes. Após o workshop, questionários e entrevistas mostraram que os meninos mantiveram o seu interesse, enquanto as meninas tiveram um aumento expressivo no seu interesse. Após o workshop, verificou-se todos avaliaram que o trabalho de programação não era uma atividade tão difícil. O workshop teve um impacto maior sobre as opiniões das meninas sobre programação, fazendo com que elas percebessem que programar pode ser divertido e que essa área não é estrita para homens.

An (2016), examinou o impacto do ensino de programação utilizando a metodologia de learning-by-making e compartilhamento de artefatos. Durante nove semanas, 12 alunos ( 8 meninos e 4 meninas) de uma escola tiveram aulas diárias sobre programação de jogos. Os participantes eram estimulados a criar soluções de design em conjunto. O processo de design envolveu o estudo de quatro eventos históricos relacionados à revolução comunista chinesa, guerra fria, guerra da Coréia e a guerra do Vietnã. Os alunos que participaram eram gamers e a maioria não tinha conhecimento prévio em programação. No início os participantes relataram que não eram criativos, porém durante o curso foi verificado que eles tiveram ideias interessantes. Entretanto, muitas ideias não eram factíveis com o Scratch. Os participantes relataram que construir jogos educativos era chato e desinteressante. Dos doze participantes, somente um relatou que não gostaria de seguir a carreira de programador de jogos. Assim, o autor sugere que expor os jovens a diversas situações pode colaborar com o processo de decisão de profissão.

Nas publicações encontradas, todas realizaram um workshop para ensinar Scratch e realizaram questionários. Com excessão de Webb e Rosson (2013), nos outros tanto 
V Congresso Brasileiro de Informática na Educação (CBIE 2016)

Anais do XXVII Simpósio Brasileiro de Informática na Educação (SBIE 2016)

meninos quanto meninas participaram do workshop. Para Corston e Colman (1996), proporcionar ambientes de discussão e aprendizagem em computação, exclusivo para mulheres pode produzir melhores níveis de confiança nelas. Essa estratégia também evita a criação de um hostil para as mulheres e elas podem se expressar e desenvolver suas habilidades melhor. Além do mais, muitas mulheres ainda convivem com o pensamento de que os homens são melhores nessas áreas e isso pode ser intimidador para elas [Konak 2016 e Spertus 1997]. Sobre a metodologia de ensino, o único a utilizar uma abordagem consagrada e avaliar seus impactos foram Webb e Rosson (2013). Conhecer e utilizar uma metodologia de ensino tem seu impacto no sucesso do processo de ensino-aprendizagem dos alunos (2013). Em relação a quantidade de dias utilizado, quatro estudos relatam workshops de curta duração, não havendo um acompanhamento a longo prazo, mentoria e não há relato de criação de grupos de discussão para troca de conhecimento. Sendo que pesquisas mostram que programas de mentoria podem melhorar a taxa de recrutamento e retenção de mulheres em ciência da computação [Spertus 1997]. Além disso, a internet pode ser utilizada para mediar essa comunicação, como realizado em [Bennett et al. 2006]. Todos os artigos tinham como motivação a inserção de mulheres na carreira de tecnologia da informação, porém nenhum verificou se o engajamento perdurou. A escolha da linguagem de programação Scratch se deu uma vez que não requer conhecimento prévio de programação é considerada muito fácil e ágil de utilizar. Essa facilidade já tinha sido evidenciada em outros estudos, como em [Freitas et al. 2015].

\section{Conclusão}

Neste trabalho foi realizado um mapeamento sistemático sobre o uso do Scratch no ensino de programação para meninas em fase de escolha de profissão, com intuito de incentivá-las para seguir uma carreira em computação. Mapeamentos são relevantes por apresentarem um cenário da área em estudo, auxiliando estudos mais aprofundados e específicos.

Visando responder as perguntas de pesquisa, após a seleção de artigos sobre o uso do Scratch para ensino de programação no ensino médio, foram encontradas 601 publicações, respondendo assim a pergunta de pesquisa (RQ1). Com isso, verificou-se que o uso do Scratch, como ferramenta de ensino, é um tema recente e bastante motivador. Quando verificado sobre o incentivo para mulheres na computação, foram encontrados somente 5 artigos (RQ2). Os artigos constataram indícios que o ensino da linguagem Scratch pode facilitar o aprendizado em computação para leigos. Com isso, pode auxiliar a motivar as mulheres a descontruir certos estigmas e incentivá-las a seguir uma carreira na computação. Sobre as abordagens utilizadas pelos estudos encontrados para incentivar mulheres na computação utilizando a linguagem Scratch, nota-se que os artigos encontrados adotaram a realização de workshops (RQ3). No entanto, verifica-se que somente essa abordagem é insatisfatória. Por isso, mais estudos são necessários para explorar múltiplas abordagens, como por exemplo palestras e fóruns.

Esse mapeamento mostrou que expor meninas a programação por meio da linguagem de programação Scratch, pode ter um impacto positivo na escolha da profissão. Entretanto, nenhum dos estudos encontrados verificaram se as meninas mantiveram o entusiasmo à longo prazo. Por mais que existem várias iniciativas de incentivo para o ensino de programação e para atrair mais mulheres à computação, poucos estudos têm sido realizados para melhorar a eficácia nessa área multidisciplinar [Freitas et al. 2015].

Em relação as limitações deste estudo, visando apresentar sugestões para futuros estudos relacionados. Sobre a string de busca, vale ressaltar que o processo para 
V Congresso Brasileiro de Informática na Educação (CBIE 2016)

Anais do XXVII Simpósio Brasileiro de Informática na Educação (SBIE 2016)

construção foi iterativo. Além disso, houve a necessidade de refinamentos na estrutura para se adequar à cada máquina de busca. Em relação a quantidade de estudos, ressaltase que outras fontes de dados podem ser adicionadas à pesquisa, como livros, artigos que não são peer-reviewed e que não estão publicados em bases científicas. No entanto, por mais que estas fontes poderiam colaborar, elas não atendiam os critérios de fontes do protocolo. Além disso, algumas fontes podem ter acesso restrito ou não-gratuito.

Por mais que vários artigos discutam o papel e a importância das mulheres na computação, verificou-se durante a realização desse mapeamento que é preciso explorar mais a questão do ensino de computação nas escolas. Acredita-se que o número de artigos relacionados ao tema, Scratch para incentivar meninas na computação é pequeno pelo fato que a linguagem Scratch foi lançada em 2007 e começou a ser utilizada de forma abrangente na educação em 2009. Além disso, o ensino de programação ainda não faz parte dos parâmetros curriculares em vários países. Nesse sentido, os Estados Unidos têm sido referência e com iniciativas como o Code.org que tem estimulado o ensino de programação nas escolas, por meio de uma plataforma online, colaborativa e interativa.

Como sugestão para trabalhos futuros na área, destaca-se a necessidade de haver um melhor detalhamento da metodologia empregada para a construção das atividades realizadas e a realização de um acompanhamento por meio de grupo de discussões online para que haja trocas de experiências e colaboração em projetos individuais. Observa-se também que pode ser interessante verificar se aprendizagem exclusiva a meninas para poder produzir melhores níveis de confiança e autoeficácia nelas, e uma possível comparação com turmas não-exclusivas.

\section{Referências}

An, Y. A case study of educational computer game design by middle school students. In: Educational Technology Research and Development. pp. 1-17, 2016.

Bennett, D., et al. Telementoring: Designing on-line mentoring environments for high school women in science and technical courses. Women in Engineering ProActive Network, 2006.

Bolfer, M. Reflexões sobre prática docente: estudo de caso sobre formação continuada de professores universitários. Tese de Doutorado. Universidade Metodista de Piracicaba.

Borg, A. Computing 2002: democracy, education, and the future. In: ACM SIGCSE Bulletin. v. 34, n. 2, pp. 13-14, 2002.

Centro de Informática UFPB. Acessado em Maio/2016. http://ci.ufpb.br/projetos-de-extensao/

Corston, R., Colman, A. M.. Gender and social facilitation effects on computer competence and attitudes toward computers. In: Journal of educational computing research. v. 14, n. 2, p. 171-183, 1996.

Feldhausen, R., Bell, S., and Andresen, D.. Minimum Time, Maximum Effect: Introducing Parallel Computing in CS0 and STEM Outreach Activities Using Scratch. In: Proceedings of the 2014 Annual Conference on Extreme Science and Engineering Discovery Environment (XSEDE '14). ACM, NY, USA, Article 75, 7 p., 2014.

Gordon, V. N.. The undecided student: A developmental perspective. Personnel and Guidance Journal, v. 59, n.7, pp. 433-439, 1981.

Gürer, D., and Camp, T. An ACM-W literature review on women in computing. In: $A C M$ SIGCSE Bulletin v. 34, n. 2, pp. 121-127, 2002. 
V Congresso Brasileiro de Informática na Educação (CBIE 2016)

Anais do XXVII Simpósio Brasileiro de Informática na Educação (SBIE 2016)

Huber, B. R., Scaglion, R. Gender differences in computer education: A Costa Rican case study. In: Journal of Educational Computing Research, n. 3, pp. 271-304, 1995.

Kitchenham, B., et al. Systematic literature reviews in software engineering-a systematic literature review. In: Information and software technology v. 51, n. 1, 7-15, 2009.

Klawe, M. Girls, boys, and computers. In: ACMSIGCSE Bulletin, v. 34, n. 2, pp. 16-17, 2002.

Konak, J.. Programming for girls. 2016 IEEE. In: Integrated STEM Education Conference (ISEC). IEEE, 2016.

Krendl, K. A., Broihier, M. C., and Fleetwood, C. Children and Computers: Do SexRelated Differences Persist? In: Journal of Communication, pp. 85-93, 1989.

Lombardi, M. R.. Engenheiras brasileiras: inserção e limites de gênero no campo profissional. Cadernos de Pesquisa n. 127, Fundação Carlos Chagas, pp. 173-202, 2006.

Mackey, A., Gass, S. Common data collection measures. Second language research: methodology and design. Mahwah: Lawrence Erlbaum. pp. 43-99, 2005.

Maia, M. M.. Gender obstacles and the presence of women in Brazilian Computing graduate courses. Cadernos Pagu, n. 46, pp. 223-244, 2016.

Martins, A. R. Q.. Usando o Scratch Para Potencializar o Pensamento Criativo em Crianças do Ensino Fundamental. Tese de Doutorado. Universidade de Passo Fundo. 2012.

Mota, F. P., Adamatti, D. F.. Programming teaching in high schools: An analysis based on the discourse of collective subject. In: FIE Conference, IEEE, 2015.

Petersen, K., et al. Systematic mapping studies in software engineering. In: 12th international conference on evaluation and assessment in software engineering. 2008.

Ramos, N., Freitas, C., Avila, S., Costa, P. D. P., Testoni, V., \& Borin, J. F. Ensino de Programação para Alunas de Ensino Médio: Relato de uma Experiência. 2015.

Saeli, M., Perrenet, J., Jochems, W. M. G., Zwaneveld, B. Teaching programming in secondary school: a pedagogical content knowledge perspective. In: Informatics in Education, v.10, n.1, 2011.

Spertus, E. Wit Helps Women in Computer Science Combat Ignorance. In: SIGCSE Bulletin, v. 34, n. 2, 2002. Reprinted from Women in Higher Education, Maio, 1997.

Sturm, D., and Moroh, M. Encouraging Enrollment and Retention of Women in Computer Science Classes. 1994.

Webb, H., Rosson, M. B. Using scaffolded examples to teach computational thinking concepts. In: Proceeding of the 44th ACM SIGCSE. ACM, NY, USA, pp. 95-100, 2013.

Wohlin, C. Guidelines for snowballing in systematic literature studies and a replication in software engineering. In: Proceedings of the 18th Int. Conf. on EASE. ACM, 2014.

Wulff, M. B., Steitz, J. A.. A path model of the relationship between career indecision, androgyny, self-efficacy and self-esteem. Perceptual and Motor Skills, pp. 935-940, 1999.

Zamboni, A., Fabbri, S. StArt uma ferramenta computacional de apoio à revisão sistemática. In: Proceedings of CBSoft'10, Salvador, Brazil. 2010. 